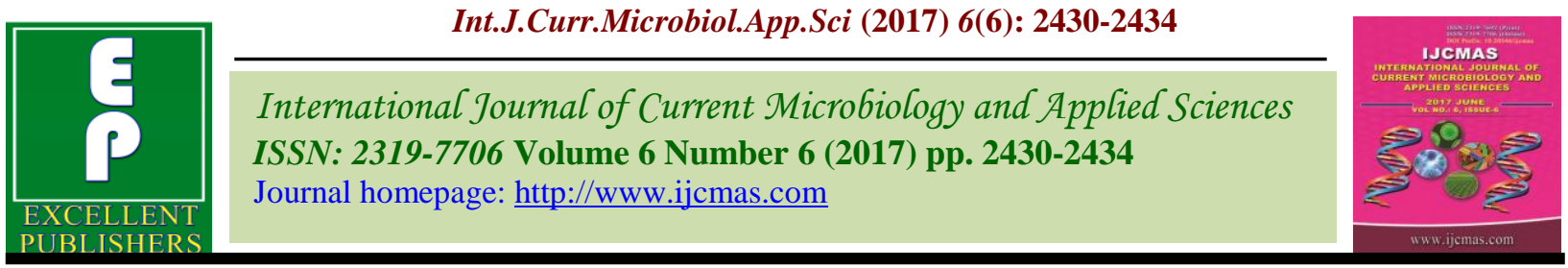

Original Research Article https://doi.org/10.20546/ijcmas.2017.606.288

\title{
Estimation of Soil Macro Arthropods in agriculture, Horticulture and Forest Ecosystems
}

\author{
Salavuddin Mohammad", Bontha Rajasekar and R.K. Patil
}

Department of Agricultural Entomology, University of Agricultural Sciences, Dharwad - 580 005, Karnataka, India

*Corresponding author

\section{A B S T R A C T}

\begin{tabular}{l} 
Ke y w o r d s \\
$\begin{array}{l}\text { Soil macro } \\
\text { arthropods, Eco } \\
\text { system, Forest, } \\
\text { Horticulture and } \\
\text { Pitfall traps. }\end{array}$ \\
\hline Article Info \\
\hline $\begin{array}{l}\text { Accepted: } \\
\text { 29 May } 2017 \\
\text { Available Online: } \\
\text { 10 June } 2017\end{array}$ \\
\hline
\end{tabular}

Keywords

Soil macro arthropods, Eco system, Forest Horticulture and . 10 June 2017
A field investigation was undertaken during Kharif-2013 to estimate the population of soil macro arthropods in agriculture, horticulture and forest ecosystems. Soil macro fauna it includes ants, carabids, centipedes, millipedes and others. Among the different eco systems, forest ecosystem recorded more population of soil macro arthropods (214.67/15 pitfall traps) followed by horticulture ecosystem (119.71/15 pitfall traps) and agriculture ecosystem (145.67/15 pitfall traps). Irrespective of farming systems the population of soil macro arthropods gradually increased from first fortnight of June (116.00/15pitfall traps) and reached to peak population during second fortnight of August (225.44/15pitfall traps) and gradually decreased to least population during second fortnight of September (182.33/15pitfall traps).

\section{Introduction}

Soil is the most valuable resource, since this is essential to the entire plant life on which animal and human life depends. Soil contains wide assortment of organic, inorganic substances, water and gases thus provide a unique medium for growth and development of micro-organisms and soil invertebrates. In general, soil invertebrates are classified according to their size into three classes' viz., micro fauna, meso fauna and macro fauna. Soil ecosystem services are reliant upon soil fauna which provide benefit to human population. They support most production systems through soil formation, nutrient cycling and primary production and also participate in provision of regulation services like climate regulation and detoxification. Without soil organisms, the soil would be a sterile medium that could not sustain crop production. Soil biota provides essential benefits for the functioning of agro ecosystems which are important for the long term sustainability of agriculture. They support essential soil processes and play a key role in maintaining the soil quality that is necessary for crop productivity. Soil organisms help to create and maintain beneficial soil structure and decomposing crop residues so that valuable nutrients are released for plant growth and contribute to soil carbon storage by mixing organic materials with mineral soil. Long-term and 
large number of soil inorganic fertilizer applications can affect negatively on soil fertility, soil biodiversity and crop products quality (Gruzdeva et al., 2007). By, considering the increasing importance of organic agriculture, soil organic manure applications are desirable and recommended. The effect deriving from organic manure applications includes numerous benefits resulting in an improvement of physical, biological and chemical soil properties, i.e. porosity, aggregates stability, water exchange and fertility (Tester, 1990). Heavy agricultural reliance on synthetic-chemical fertilizers and pesticides is having deleterious impacts on soil arthropods and the environment. With this background studies on soil arthropods in different farming systems were taken up.

\section{Materials and Methods}

The experiment was conducted during kharrif 2013 at UAS Dharwad campus. The experiment was conducted with pitfall traps to know population of soil macro fauna which are moving above the ground. Pitfall traps are helpful in collecting the surface moving arthropods these are helpful to know the count of surface moving soil macro arthropods. Fifteen pitfall traps were placed in each field of agriculture, horticulture and forest ecosystems for collection of soil macro fauna. The trap consists of plastic cups $(5 \mathrm{~cm}$ in diametre $7 \mathrm{~cm}$ in height). The traps were buried in the ground with their rim leveled with the soil surface to facilitate wandering fauna to fall inside. Each cup was filled with $50 \mathrm{ml}$ of $75 \%$ ethyl alcohol as killing agent with bit of glycerol to prevent the evaporation of alcohol. Observations were taken with fifteen days interval starting from June till the harvest of the crops. Population of each group was recorded. Further these were separated as different taxonomic groups and preserved for further needful. Data were subjected to statistical analysis by two ways ANOVA.

\section{Results and Discussion}

Forest ecosystem recorded significantly highest population of centipedes (15.38/15 pitfall traps), spiders (15.83/15 pitfall traps), others (17.98 /15 pitfall traps) and total macro arthropods (177.55/15 pitfall traps) it was followed by horticultural ecosystem and least population of centipedes $(9.86 / 15$ pitfall traps), spiders ( $9.95 / 15$ pitfall traps), others (cockroaches, crickets and grasshoppers) (12.05 /15 pitfall traps) and total macro arthropods (116.33/15 pitfall traps) were observed in agricultural ecosystem (Table 1). Irrespective of forest, horticultural and agricultural ecosystems population of centipedes gradually increased from first fortnight of June (9.00/15 pitfall traps) and attained maximum population during first fortnight of August (21.11/15 pitfall traps) then gradually decreased and attained minimum population during first fortnight of December (5.33/15 pitfall traps) (Table 1). Interaction effect was found non-significant with respect to centipedes' population between different ecosystems and dates of collection. Irrespective of forest, horticultural and agricultural ecosystems population of spiders gradually increased from first fortnight of June (11.00/15 pitfall traps) and attained maximum population during second fortnight of August (19.11/15 pitfall traps) then gradually decreased and attained minimum population during second fortnight of December (6.88/15 pitfall traps) (Table 1). Interaction effect was significant between different ecosystems and dates of collection with respective to spiders population. Significantly highest population of spiders population was recorded in forest ecosystem during second fortnight of August (23.00/15 pitfall traps) and least population recorded in agricultural ecosystem during December second fortnight (5.00/15 pitfall traps) (Table $1)$. 
Table.1 Population of soil macro arthropods in agricultural, horticultural and forest ecosystems during 2013 (Per 15 pitfall traps)

\begin{tabular}{|c|c|c|c|c|c|c|c|c|c|c|c|c|c|c|c|c|}
\hline \multirow{2}{*}{$\begin{array}{c}\begin{array}{c}\text { Month of } \\
\text { collection(MC) }\end{array} \\
\begin{array}{c}\text { Ecosystems } \\
(\text { ES })\end{array} \\
\end{array}$} & \multicolumn{4}{|c|}{ Centipedes (No./15 pitfall traps) } & \multicolumn{4}{|c|}{ Spiders (No./15 pitfall traps) } & \multicolumn{4}{|c|}{$\begin{array}{c}\text { Others(cockroach, crickets and } \\
\text { grasshoppers) (No./15 pitfall traps) }\end{array}$} & \multicolumn{4}{|c|}{$\begin{array}{l}\text { Total macro arthropods (No./15 pitfall } \\
\text { traps) }\end{array}$} \\
\hline & $\mathbf{A E}$ & HE & FE & Mean & $\mathbf{A E}$ & HE & FE & Mean & $\mathbf{A E}$ & HE & FE & Mean & $\mathbf{A E}$ & HE & FE & Mean \\
\hline June I fortnight & $\begin{array}{r}6.00 \\
(2.55) \\
\end{array}$ & $\begin{array}{r}9.00 \\
(3.08) \\
\end{array}$ & $\begin{array}{r}12.00 \\
(3.54)\end{array}$ & $9.00 \mathbf{j}$ & $\begin{array}{r}7.67 \\
(2.86) \\
\end{array}$ & $\begin{array}{r}11.00 \\
(3.39)\end{array}$ & $\begin{array}{r}14.33 \\
(3.85)\end{array}$ & $11.00 \mathrm{~h}$ & $\begin{array}{r}10.67 \\
(3.34) \\
\end{array}$ & $\begin{array}{r}14.00 \\
(3.81) \\
\end{array}$ & $\begin{array}{r}18.67 \\
(4.38) \\
\end{array}$ & $14.44 \mathrm{~g}$ & $\begin{array}{l}83.67 \\
(9.17)\end{array}$ & $\begin{array}{r}116.00 \\
(10.79) \\
\end{array}$ & $\begin{array}{r}148.33 \\
(12.20) \\
\end{array}$ & $116.00 \mathrm{k}$ \\
\hline $\begin{array}{c}\text { June II } \\
\text { fortnight }\end{array}$ & $\begin{array}{r}10.00 \\
(3.24) \\
\end{array}$ & $\begin{array}{r}12.67 \\
(3.63) \\
\end{array}$ & $\begin{array}{r}16.00 \\
(4.06) \\
\end{array}$ & $12.88 \mathrm{~g}$ & $\begin{array}{r}8.33 \\
(2.97) \\
\end{array}$ & $\begin{array}{r}11.67 \\
(3.49) \\
\end{array}$ & $\begin{array}{r}15.67 \\
(4.02) \\
\end{array}$ & $11.88 \mathrm{~g}$ & $\begin{array}{r}13.67 \\
(3.76) \\
\end{array}$ & $\begin{array}{r}18.67 \\
(4.38) \\
\end{array}$ & $\begin{array}{r}22.67 \\
(4.81) \\
\end{array}$ & 18.33d & $\begin{array}{r}104.33 \\
(10.24) \\
\end{array}$ & $\begin{array}{r}134.00 \\
(11.60) \\
\end{array}$ & $\begin{array}{r}161.33 \\
(12.72) \\
\end{array}$ & 133.22i \\
\hline July I fortnight & $\begin{array}{r}12.67 \\
(3.63) \\
\end{array}$ & $\begin{array}{r}15.00 \\
(3.94) \\
\end{array}$ & $\begin{array}{r}17.67 \\
(4.26) \\
\end{array}$ & 15.11d & $\begin{array}{r}13.00 \\
(3.67) \\
\end{array}$ & $\begin{array}{r}15.67 \\
(4.02) \\
\end{array}$ & $\begin{array}{r}20.00 \\
(4.53) \\
\end{array}$ & $16.22 \mathrm{c}$ & $\begin{array}{r}14.00 \\
(3.81) \\
\end{array}$ & $\begin{array}{r}18.00 \\
(4.30) \\
\end{array}$ & $\begin{array}{r}20.00 \\
(4.53) \\
\end{array}$ & $17.33 \mathrm{e}$ & $\begin{array}{r}123.00 \\
(11.11) \\
\end{array}$ & $\begin{array}{r}148.33 \\
(12.20) \\
\end{array}$ & $\begin{array}{r}170.33 \\
(13.07) \\
\end{array}$ & $147.22 \mathrm{~g}$ \\
\hline July II fortnight & $\begin{array}{l}11.67 \\
(3.49) \\
\end{array}$ & $\begin{array}{r}14.00 \\
(3.81) \\
\end{array}$ & $\begin{array}{r}18.00 \\
(4.30) \\
\end{array}$ & $14.55 \mathrm{e}$ & $\begin{array}{r}14.00 \\
(3.81)\end{array}$ & $\begin{array}{r}17.00 \\
(4.18) \\
\end{array}$ & $\begin{array}{r}18.67 \\
(4.38) \\
\end{array}$ & $16.55 \mathrm{~b}$ & $\begin{array}{l}18.67 \\
(4.38) \\
\end{array}$ & $\begin{array}{r}22.00 \\
(4.74)\end{array}$ & $\begin{array}{r}24.00 \\
(4.95) \\
\end{array}$ & $21.55 b$ & $\begin{array}{r}142.00 \\
(11.94) \\
\end{array}$ & $\begin{array}{r}170.00 \\
(13.06) \\
\end{array}$ & $\begin{array}{r}189.67 \\
(13.79) \\
\end{array}$ & $167.22 \mathrm{e}$ \\
\hline Aug I fortnight & $\begin{array}{r}17.67 \\
(4.26) \\
\end{array}$ & $\begin{array}{r}21.00 \\
(4.64) \\
\end{array}$ & $\begin{array}{r}24.67 \\
(5.02) \\
\end{array}$ & 21.11a & $\begin{array}{r}12.67 \\
(3.63) \\
\end{array}$ & $\begin{array}{r}16.00 \\
(4.06) \\
\end{array}$ & $\begin{array}{r}20.67 \\
(4.60) \\
\end{array}$ & $16.44 \mathrm{~b}$ & $\begin{array}{r}19.67 \\
(4.49) \\
\end{array}$ & $\begin{array}{r}21.67 \\
(4.71) \\
\end{array}$ & $\begin{array}{r}23.00 \\
(4.85) \\
\end{array}$ & $21.44 b$ & $\begin{array}{r}170.33 \\
(13.07) \\
\end{array}$ & $\begin{array}{r}213.00 \\
(14.61) \\
\end{array}$ & $\begin{array}{r}253.67 \\
(15.94) \\
\end{array}$ & 212.33c \\
\hline Aug II fortnight & $\begin{array}{r}16.00 \\
(4.06)\end{array}$ & $\begin{array}{r}18.67 \\
(4.38)\end{array}$ & $\begin{array}{r}22.67 \\
(4.81) \\
\end{array}$ & 19.11b & $\begin{array}{r}14.67 \\
(3.89)\end{array}$ & $\begin{array}{r}19.67 \\
(4.49) \\
\end{array}$ & $\begin{array}{r}23.00 \\
(4.85)\end{array}$ & $19.11 \mathrm{a}$ & $\begin{array}{r}16.67 \\
(4.14)\end{array}$ & $\begin{array}{r}20.00 \\
(4.53)\end{array}$ & $\begin{array}{r}24.67 \\
(5.02)\end{array}$ & $20.44 c$ & $\begin{array}{r}179.00 \\
(13.40)\end{array}$ & $\begin{array}{r}228.33 \\
(15.13) \\
\end{array}$ & $\begin{array}{r}269.00 \\
(16.42) \\
\end{array}$ & 225.44a \\
\hline Sep I fortnight & $\begin{array}{r}15.67 \\
(4.02)\end{array}$ & $\begin{array}{r}18.67 \\
(4.38)\end{array}$ & $\begin{array}{r}20.67 \\
(4.60)\end{array}$ & $18.33 c$ & $\begin{array}{r}13.00 \\
(3.67)\end{array}$ & $\begin{array}{r}15.67 \\
(4.02)\end{array}$ & $\begin{array}{r}19.00 \\
(4.42)\end{array}$ & $15.88 d$ & $\begin{array}{r}18.67 \\
(4.38)\end{array}$ & $\begin{array}{l}21.33 \\
(4.67)\end{array}$ & $\begin{array}{r}25.67 \\
(5.12)\end{array}$ & 21.88a & $\begin{array}{r}176.33 \\
(13.30)\end{array}$ & $\begin{array}{r}218.00 \\
(14.78)\end{array}$ & $\begin{array}{r}247.67 \\
(15.75)\end{array}$ & 214.00b \\
\hline Sep II fortnight & $\begin{array}{r}11.67 \\
(3.49) \\
\end{array}$ & $\begin{array}{r}14.00 \\
(3.81) \\
\end{array}$ & $\begin{array}{r}17.00 \\
(4.18) \\
\end{array}$ & $14.22 \mathrm{f}$ & $\begin{array}{r}11.67 \\
(3.49) \\
\end{array}$ & $\begin{array}{r}14.00 \\
(3.81) \\
\end{array}$ & $\begin{array}{r}17.67 \\
(4.26) \\
\end{array}$ & $14.44 \mathrm{e}$ & $\begin{array}{r}13.67 \\
(3.76) \\
\end{array}$ & $\begin{array}{r}16.00 \\
(4.06) \\
\end{array}$ & $\begin{array}{r}19.67 \\
(4.49) \\
\end{array}$ & $16.44 f$ & $\begin{array}{r}145.67 \\
(12.09) \\
\end{array}$ & $\begin{array}{r}186.67 \\
(13.68) \\
\end{array}$ & $\begin{array}{r}214.67 \\
(14.67) \\
\end{array}$ & 182.33d \\
\hline Oct I fortnight & $\begin{array}{l}10.00 \\
(3.24) \\
\end{array}$ & $\begin{array}{r}13.00 \\
(3.67) \\
\end{array}$ & $\begin{array}{r}16.00 \\
(4.06) \\
\end{array}$ & $13.00 \mathrm{~g}$ & $\begin{array}{r}9.67 \\
(3.19) \\
\end{array}$ & $\begin{array}{r}12.67 \\
(3.63) \\
\end{array}$ & $\begin{array}{r}15.00 \\
(3.94) \\
\end{array}$ & $12.44 f$ & $\begin{array}{r}12.00 \\
(3.54) \\
\end{array}$ & $\begin{array}{r}13.00 \\
(3.67) \\
\end{array}$ & $\begin{array}{r}15.00 \\
(3.94) \\
\end{array}$ & 13.33h & $\begin{array}{r}124.33 \\
(11.17) \\
\end{array}$ & $\begin{array}{r}160.00 \\
(12.67) \\
\end{array}$ & $\begin{array}{r}185.00 \\
(13.62) \\
\end{array}$ & $156.44 f$ \\
\hline Oct II fortnight & $\begin{array}{r}8.00 \\
(2.92) \\
\end{array}$ & $\begin{array}{r}11.00 \\
(3.39) \\
\end{array}$ & $\begin{array}{r}13.00 \\
(3.67) \\
\end{array}$ & $10.66 \mathrm{~h}$ & $\begin{array}{r}8.00 \\
(2.92) \\
\end{array}$ & $\begin{array}{r}11.00 \\
(3.39) \\
\end{array}$ & $\begin{array}{r}13.67 \\
(3.76) \\
\end{array}$ & 10.88hi & $\begin{array}{r}10.00 \\
(3.24) \\
\end{array}$ & $\begin{array}{r}12.00 \\
(3.54) \\
\end{array}$ & $\begin{array}{r}14.00 \\
(3.81) \\
\end{array}$ & $12.00 \mathrm{i}$ & $\begin{array}{r}104.67 \\
(10.26)\end{array}$ & $\begin{array}{r}141.33 \\
(11.91)\end{array}$ & $\begin{array}{r}162.33 \\
(12.76)\end{array}$ & 136.11h \\
\hline Nov I fortnight & $\begin{array}{r}7.00 \\
(2.74)\end{array}$ & $\begin{array}{r}10.00 \\
(3.24)\end{array}$ & $\begin{array}{r}12.67 \\
(3.63)\end{array}$ & $9.88 \mathrm{i}$ & $\begin{array}{r}6.67 \\
(2.68) \\
\end{array}$ & $\begin{array}{r}9.67 \\
(3.19)\end{array}$ & $\begin{array}{r}12.00 \\
(3.54)\end{array}$ & $9.44 j$ & $\begin{array}{r}7.00 \\
(2.74)\end{array}$ & $\begin{array}{r}9.00 \\
(3.08)\end{array}$ & $\begin{array}{r}11.00 \\
(3.39)\end{array}$ & $9.00 \mathrm{j}$ & $\begin{array}{r}89.00 \\
(9.46) \\
\end{array}$ & $\begin{array}{r}122.00 \\
(11.07) \\
\end{array}$ & $\begin{array}{r}145.33 \\
(12.08) \\
\end{array}$ & 118.78j \\
\hline Nov II fortnight & $\begin{array}{r}5.00 \\
(2.35) \\
\end{array}$ & $\begin{array}{r}7.00 \\
(2.74) \\
\end{array}$ & $\begin{array}{r}9.00 \\
(3.08) \\
\end{array}$ & $7.00 \mathrm{k}$ & $\begin{array}{r}8.00 \\
(2.92) \\
\end{array}$ & $\begin{array}{r}10.67 \\
(3.34) \\
\end{array}$ & $\begin{array}{r}13.67 \\
(3.76) \\
\end{array}$ & $10.77 \mathbf{i}$ & $\begin{array}{r}6.00 \\
(2.55) \\
\end{array}$ & $\begin{array}{r}8.67 \\
(3.03) \\
\end{array}$ & $\begin{array}{r}12.00 \\
(3.54) \\
\end{array}$ & $8.88 \mathrm{j}$ & $\begin{array}{r}73.67 \\
(8.61) \\
\end{array}$ & $\begin{array}{l}106.33 \\
(10.34) \\
\end{array}$ & $\begin{array}{l}130.33 \\
(11.44) \\
\end{array}$ & 103.44l \\
\hline Dec I fortnight & $\begin{array}{r}3.00 \\
(1.87) \\
\end{array}$ & $\begin{array}{r}5.00 \\
(2.35) \\
\end{array}$ & $\begin{array}{r}8.00 \\
(2.92) \\
\end{array}$ & $5.33 \mathrm{~m}$ & $\begin{array}{r}7.00 \\
(2.74) \\
\end{array}$ & $\begin{array}{r}11.00 \\
(3.39) \\
\end{array}$ & $\begin{array}{r}9.67 \\
(3.19) \\
\end{array}$ & $9.22 \mathrm{k}$ & $\begin{array}{r}5.00 \\
(2.35) \\
\end{array}$ & $\begin{array}{r}7.67 \\
(2.86) \\
\end{array}$ & $\begin{array}{r}11.67 \\
(3.49) \\
\end{array}$ & $8.11 k$ & $\begin{array}{r}63.67 \\
(8.01) \\
\end{array}$ & $\begin{array}{l}93.67 \\
(9.70) \\
\end{array}$ & $\begin{array}{l}111.00 \\
(10.56) \\
\end{array}$ & $89.44 \mathrm{~m}$ \\
\hline Dec II fortnight & $\begin{array}{r}3.67 \\
(2.04) \\
\end{array}$ & $\begin{array}{r}5.67 \\
(2.48) \\
\end{array}$ & $\begin{array}{r}8.00 \\
(2.92) \\
\end{array}$ & 5.781 & $\begin{array}{r}5.00 \\
(2.35)\end{array}$ & $\begin{array}{r}7.00 \\
(2.74) \\
\end{array}$ & $\begin{array}{r}8.67 \\
(3.03) \\
\end{array}$ & $6.88 \mathrm{~g}$ & $\begin{array}{r}3.00 \\
(1.87)\end{array}$ & $\begin{array}{r}6.33 \\
(2.61) \\
\end{array}$ & $\begin{array}{r}9.67 \\
(3.19) \\
\end{array}$ & 6.331 & $\begin{array}{l}49.00 \\
(7.04) \\
\end{array}$ & $\begin{array}{r}78.33 \\
(8.88) \\
\end{array}$ & $\begin{array}{r}97.00 \\
(9.87) \\
\end{array}$ & 74.78n \\
\hline Mean & $9.86 \mathrm{c}$ & $12.48 b$ & $15.38 \mathrm{a}$ & 12.57 & $9.95 \mathrm{c}$ & 13.05b & $15.83 \mathrm{a}$ & 12.94 & $12.05 c$ & $14.88 b$ & $17.98 a$ & 14.97 & $116.33 a$ & 151.14b & $177.55 \mathrm{c}$ & 148.34 \\
\hline & \multicolumn{2}{|c|}{ S.Em. \pm} & \multicolumn{2}{|c|}{$\mathrm{CD}(\mathbf{P}=0.05)$} & \multicolumn{2}{|c|}{ S.Em. \pm} & \multicolumn{2}{|c|}{$\mathrm{CD}(\mathrm{P}=0.05)$} & \multicolumn{2}{|c|}{ S.Em. \pm} & \multicolumn{2}{|c|}{$\mathrm{CD}(\mathrm{P}=0.05)$} & \multicolumn{2}{|c|}{ S.Em. \pm} & \multicolumn{2}{|c|}{$\mathrm{CD}(\mathrm{P}=0.05)$} \\
\hline $\begin{array}{c}\text { Farming } \\
\text { Systems (FS) }\end{array}$ & \multicolumn{2}{|c|}{0.02} & \multicolumn{2}{|c|}{0.06} & \multicolumn{2}{|c|}{0.02} & \multicolumn{2}{|c|}{0.05} & \multicolumn{2}{|c|}{0.02} & \multicolumn{2}{|c|}{0.05} & \multicolumn{2}{|c|}{0.01} & \multicolumn{2}{|c|}{0.02} \\
\hline $\begin{array}{c}\text { Date of } \\
\text { collection (DC) }\end{array}$ & \multicolumn{2}{|c|}{0.05} & \multicolumn{2}{|c|}{0.13} & \multicolumn{2}{|c|}{0.04} & \multicolumn{2}{|c|}{0.11} & \multicolumn{2}{|c|}{0.04} & \multicolumn{2}{|c|}{0.11} & \multicolumn{2}{|c|}{0.01} & \multicolumn{2}{|c|}{0.04} \\
\hline FSXDC & \multicolumn{2}{|c|}{0.08} & $\mathrm{~N}$ & & & 07 & 0. & 20 & & & 0. & & 0. & & 0. & \\
\hline
\end{tabular}

FE - Forest ecosystem HE - Horticulture ecosystem AE - Agriculture Ecosystem Figures in the parenthesis are $\sqrt{ } \mathrm{x}+05$ transformed values NS - Non-significant 
Irrespective of forest, horticultural and agricultural ecosystems population of total macro arthropods gradually increased from first fortnight of June (116.00/15 pitfall traps) and attained maximum population during second fortnight of August (225.44/15 pitfall traps) then gradually decreased and attained minimum population during second fortnight of December (74.78/15 pitfall traps). The interaction effect was also found significant between different ecosystems and months of collection. Significantly highest total macro arthropods found in forest ecosystem during second fortnight of August (269.00/15 pitfall traps) and least was found in agricultural ecosystem during second fortnight of December (49.00/15 pitfall traps) (Table 1). The results indicated that forest ecosystem recorded 1.52 fold increase of macro arthropod population than agricultural ecosystem. Horticulture ecosystem recorded 1.30 fold increase of macro arthropod population compared to agricultural ecosystem. Macro arthropods include ants, carabid beetles, centipedes, spiders, scarabids, millipedes and others (grasshoppers, crickets, earwigs etc.). Most of them were predatory and their population is dependent on other meso arthropods, availability of food, prey, favorable climatic conditions and more vegetative cover. Present findings are in line with the observations of Pokarzhevskii and Krivolutskii (1997) they have reported biodiversity of soil macro fauna in a set of ecosystems. Their study indicated that decreasing trend in species number, population density and biomass in Agriecosystem compared to natural ecosystems. The main reason for the decrease appears to be a lack of availability of organic matter and essential element supply in the soils of agro-ecosystem. The results also similar results were also reported by Basu et al., (1996), Lima et al., (2010), and Kumar et al., (2013) which highlights forest ecosystem harboring more macro arthropod population compared to disturbed area. The maximum population observed in the month of August 2013 may be due to rainy season which resulted in maintaining optimum moisture content and optimum soil temperature which is favorable for growth and reproduction soil macro arthropods. The results are similar with Lima et al., (2010) who has reported highest abundance of macro-fauna in the rainy season. Agro forestry systems provide better soil chemical characteristics and increase soil invertebrate macro fauna abundance and richness. The present findings are also in line with Gondim et al., (2010) who have reported peak population in the month of August. Higher rainfall in the month of August favored higher establishment of edaphic organisms contributing to the higher diversity. Minati and Kakati (2013) who reported that maximum abundance was exhibited during rainy season followed by winter and summer. The peak population density recorded during August in natural and degraded sites. The present findings corroborate with the findings of Sanyal (1996), Tripathi et al., (2007) who recorded peak population of micro arthropods during the month of August.

\section{References}

Basu, P., Blanchart, E. and Lepage, M., 1996, Termite (Isoptera) community in the Western Ghats, South India: Influence of anthropogenic disturbance of natural vegetation. European J. Soil Bio, 32: 113-121.

Gondim, S. C., Souto, J. S., Cavalcante, L. F., Araujo, K. D. and Rodrigues, M. Q., 2010, Bio- fertilizer bovine and water salinity on soil macrofauna cultivated with yellow passion fruit.

Revista Verde de Agroecologia e Desenvolvimento Sustentavel, 5(2): 3545.

Gruzdeva, L. I., Matveeva, E. M. and Kovalenko, T. E., 2007, Changes in soil 
nematode communities under the impact of fertilizers. Eurasian Soil Sci., 40: 681-693.

Kumar, N. G., Nirmala, P., Musthak Ali, T. M. and Balakrishna, A. N., 2013, Diversity of ant species across a gradient of land use types in Western Ghats of Nilgiri Biosphere, Karnataka. $10^{\text {th }}$ National Symposium on Soil Biol. Ecol., p. 119.

Lima, S. S., De., Aquino, A. M. De., Leite, L. F. C., Velasquez, E. and Lavelle, P., 2010, Relationship between edaphic macrofauna and soil chemical attributes in different agroecosysterns. Pesquisa Agropecuaria Brasileira, 45(3): 322-331.

Minati, B. and Kakati, L. N., 2014, Population dynamics of soil Acarina in natural and degraded forest ecosystem at Pathalipam, Lakhimpur,
Assam. IOSR J. Env. Sci., 8(1): 45-50. Pokarzhevskii, A. D. and Krivolutskii, D. A., 1997, Problems of estimating and maintaining bio-diversity of soil biota in natural and agro-ecosystems: A case study of chemozern soil. Agric. Ecosys. Environ, 62(2-3): 127-133.

Sanyal, A. K., 1996, Soil arthropod population in two contrasting sites at Nadia, West Bengal. Environ. Ecol., 11(2): 346-350.

Tester, C. F., 1990, Organic amendment effects on physical and chemical properties of a sandy soil. Soil Sci. Soc. Am. J., 65: 1284-1292.

Tripathi, G. R., Kumari and Sharma, B. M., 2007, Mesofaunal bio-diversity and its importance in Thar Desert, J. Environ. Bio, 28(2):503-515.

\section{How to cite this article:}

Salavuddin Mohammad, Bontha Rajasekar and Patil, R.K. 2017. Estimation of Soil Macro Arthropods in Agriculture, Horticulture and Forest Ecosystems. Int.J.Curr.Microbiol.App.Sci. 6(6): 2430-2434. doi: https://doi.org/10.20546/ijcmas.2017.606.288 